\title{
Tarifverträge über Branchenzuschläge - eine erste Zwischenbilanz
}

\author{
HELGA SCHWITZER, DIRK SCHUMANN
}

\section{Hintergründe und Ausgangssituation}

Am 22. Mai 2012 wurde zwischen der IG Metall und dem Bundesarbeitgeberverband der Personaldienstleister (BAP) sowie dem Interessenverband Deutscher Zeitarbeitsunternehmen (iGZ) der „Tarifvertrag über Branchenzuschläge für Arbeitnehmerüberlassungen in der Metall- und Elektroindustrie“ (TV BZ ME) abgeschlossen, der am 1. November 2012 in Kraft getreten ist. Seitdem wurden acht weitere Tarifverträge über Branchenzuschläge von der IG Metall, der Industriegewerkschaft Bergbau, Chemie, Energie (IG BCE), der Eisenbahn- und Verkehrsgewerkschaft (EVG) und der Vereinten Dienstleistungsgewerkschaft (ver.di) abgeschlossen. ${ }^{1}$

Mit den Tarifverträgen über Branchenzuschläge soll die Lücke zwischen den Entgelten in der Leiharbeit und in den Einsatzbetrieben geschlossen werden. Vorausgegangen war eine intensive Auseinandersetzung der Gewerkschaften mit prekärer Beschäftigung im Allgemeinen und den Bedingungen in der Leiharbeit im Besonderen auf politischer und betrieblicher Ebene. ${ }^{2}$ Das Ergebnis dieser und vieler anderer Initiativen war ein erheblicher Druck auf die Leiharbeitsbranche, aber auch auf die Einsatzbetriebe und die politisch Handelnden. Auf der Regelungsebene führte dies allein im Bereich der IG Metall zu über 1.200 betrieblichen oder firmentariflichen Vereinbarungen, die die Arbeitsbedingungen der eingesetzten Leiharbeitsbeschäftigten und die Handlungsmöglichkeiten der Betriebsräte in den Ein- satzbetrieben verbesserten, außerdem zu Tarifverträgen in der Eisen- und Stahlindustrie. ${ }^{3}$ Mit den Tarifverträgen über Branchenzuschläge wurde nunmehr auch für Betriebe ohne solche sogenannten „Besservereinbarungen“ ein wichtiger Schritt zur gleichen Bezahlung von Leiharbeitsbeschäftigten vollzogen. Wenige Tage vor Abschluss des TV BZ ME konnte die IG Metall mit dem Tarifergebnis für die Metall- und Elektroindustrie am 19. Mai 2012 außerdem einen Tarifvertrag zur Verbesserung der Beteiligungsmöglichkeiten der Betriebsräte beim Einsatz von Leiharbeit durchsetzen („Tarifvertrag Leih- und Zeitarbeit - TV LeiZ“). ${ }^{4}$

Tarifverhandlungen in der Leiharbeit waren und sind eine zweischneidige und komplizierte Angelegenheit. Die DGB-Gewerkschaften haben mit der Novellierung des Arbeitnehmerüberlassungsgesetzes im Jahr 2003 dieses tarifpolitische Handlungsfeld besetzt. Das ursprüngliche Ziel einer adäquaten tariflichen Ausgestaltung des gesetzlichen Gleichbehandlungsgrundsatzes konnte damals bekanntermaßen nicht erreicht werden. Ein wesentlicher Grund war das Auftreten der CGZP (Tarifgemeinschaft Christlicher Gewerkschaften für Zeitarbeit und Personal-Service-Agenturen), die konkurrierende Tarifverträge zu Dumpingbedingungen abschloss. Die von der Leiharbeitsbranche befürchtete „Erpressungssituation“ (Gleichbehandlung oder Tarifvertrag) hatte sich damit schlagartig ins Gegenteil verkehrt: Die DGB-Gewerkschaften mussten sich fortan die günstigeren CGZP-Tarifverträge vorhalten lassen, Verbesserungen waren schwierig durchzusetzen. ${ }^{5}$ Tarifver-

\footnotetext{
1 Dies sind die TV BZ für die Holz- und Kunststoffverarbeitende Industrie und dieTextil- und Bekleidungsindustrie (in Kraft seit 1. April 2013), für die Chemische Industrie (seit 1. November 2012), die Kunststoffverarbeitende Industrie und die Kautschukverarbeitende Industrie (beide seit 1. Januar 2013), für den Schienenverkehrsbereich (seit 1. April 2013) sowie für die Papier, Pappe und Kunststoffe verarbeitende Industrie (ab 1. Mai 2013) und für die gewerblich Beschäftigten in der Druckindustrie (ab 1. Juli 2013).

2 Vgl. die Internetseite zur Kampagne der IG Metall: http:// www.gleichearbeit-gleichesgeld.de/.

3 Vgl. Karthaus, B. (2010): Der Kampf um die Leiharbeitslöhne hat gerade erst begonnen, in: Arbeit und Recht (AuR) 12/2010, S. 494.
}

\footnotetext{
4 Vgl. Schumann, D. (2012): Tarifverträge zur Leiharbeit in der Metall- und Elektroindustrie, in: Arbeitsrecht im Betrieb (AiB) 11/2012, S. 423; Krause, R. (2012): Neue tarifvertragliche Regeln für die Leiharbeit in der Metallindustrie, in: Neue Zeitschrift für Arbeitsrecht (NZA) 29 (15), S. 830; Ulber, D. (2013): Erweiterte Mitbestimmungsrechte des Betriebsrats durch Tarifverträge zur Leiharbeit, in: Arbeit und Recht (AuR) 3/2013, S. 114.
}

5 Z.B. konnte eine im Entgeltrahmentarifvertrag BAP/BZA 2003 festgelegte Verhandlungsverpflichtung über Branchenzuschläge in dieser Form nie umgesetzt werden. 
träge für die Leiharbeit tragen seitdem den Makel der Verschlechterung des gesetzlichen Gleichbehandlungsgebotes in sich.

Die bis 2010 bestehenden Tarifverträge des CGZP sind vom Bundesarbeitsgericht für unwirksam erklärt worden, inzwischen wurden auch die späteren Tarifverträge Ende März 2013 einvernehmlich beendet. ${ }^{6}$ Offenbar waren die Arbeitgeber nicht mehr bereit, die rechtlichen Risiken einer Anwendung der CGZP-Tarifverträge zu tragen. Diese Veränderung der Ausgangsbedingungen hat allerdings zu einer grundsätzlichen Debatte darüber geführt, ob die DGB-Gewerkschaften weiterhin Tarifverträge für die Leiharbeit abschließen oder ebenfalls das Tarifgeschäft für diesen Bereich beenden sollen, um das gesetzliche Gleichbehandlungsgebot zur Geltung zu bringen. Die aktuellen Tarifverhandlungen über Entgelte und Mantelbestimmungen in der Leiharbeit haben dieser Diskussion weiteren Auftrieb gegeben.

Aus Sicht der Gewerkschaften spricht allerdings einiges dafür, den eingeschlagenen Weg weiter zu verfolgen und die Tarifverträge in der Leiharbeit fortzuentwickeln, zumal sich durch Wegfall des CGZP die Ausgangsbedingungen für Tarifverhandlungen in der Leiharbeit deutlich verbessert haben. Neben den offenen juristischen Fragen, die sich bei einer Beendigung der Tarifverträge zur Leiharbeit stellen (insbesondere hinsichtlich der Nachwirkung) ist die Anwendung des gesetzlichen Gleichbehandlungsgebotes auch mit einer Reihe von praktischen Fragen verbunden: Zum Beispiel bliebe für den einzelnen Beschäftigten zunächst unklar, welche Ansprüche er tatsächlich besitzt, er müsste zunächst beim Entleiher Auskünfte zu den gültigen Arbeitsbedingungen einholen. Wie schwierig es in der Praxis ist, „Equal Treatment“-Ansprüche tatsächlich geltend zu machen und einzuklagen, zeigen die Verfahren im Zusammenhang mit den unwirksamen CGZP-Tarifverträgen. Jedenfalls erscheint es sehr zweifelhaft, ob es in der Praxis tatsächlich im größeren Maßstab zur Gleichbehandlung kommen würde. Allerdings muss auch den Arbeitgebern der Leiharbeit klar sein, dass eine Akzeptanz der Tarifverträge für diese Branche nur über weitere Verbesserungen erreicht werden kann. Aus Sicht der Gewerkschaften gilt es daher, die weiteren Entwicklungen kritisch im Auge zu behalten und gegenüber den Arbeitgebern deutlich zu machen, dass die Tarifverträge in der Gesamtschau am gesetzlichen Gleichbehandlungsgebot zu messen sind.

Ein Grundproblem der tarifvertraglichen Gestaltung von Leiharbeit bleibt trotz der neuen Regelungen allerdings bestehen: Zwar finden die Tarifverträge infolge der gesetzlichen Öffnungsklausel nahezu flächendeckend in der gesamten Branche Anwendung. Die Leiharbeit ist aber sehr unübersichtlich und wird von kleineren Betrieben dominiert, lediglich $13 \%$ der registrierten 18.500 Verleihbetriebe weisen 100 oder mehr Beschäftigte auf.7 Unmittelbar verbandsgebunden sind nach Angaben der beiden Arbeitgeberverbände BAP und iGZ lediglich 1.800 bzw. 2.500 Unternehmen (mit z. T. mehreren Betrieben). Hinzu kommt, dass das unmittelbarste und effektivste Instrument zur Umsetzung und
Kontrolle von Tarifverträgen, nämlich Betriebsräte, in Leiharbeitsbetrieben die absolute Ausnahme sind. Umso wichtiger ist die Rolle der Betriebsräte im Einsatzbetrieb, die in jüngster Zeit auch durch die Rechtsprechung gestärkt worden ist. ${ }^{8}$ Hinsichtlich der Überprüfung einer richtigen Anwendung der Leiharbeitstarifverträge sind aber auch die Möglichkeiten des Betriebsrats im Einsatzbetrieb, zumindest rechtlich, begrenzt. ${ }^{9}$ Für tarifgebundene Betriebe der Metallund Elektroindustrie gilt hier die Regelung des Tarifvertrages Leih- und Zeitarbeit, wonach nur Verleiher zum Einsatz kommen dürfen, die eine Vergütung mindestens in Höhe der DGB-Tarifverträge zuzüglich eines Branchenzuschlags bezahlen. Ein Verstoß hiergegen löst ein Zustimmungsverweigerungsrecht des Betriebsrates aus..$^{10}$ Ansonsten bleibt es allerdings bei einer individualrechtlichen Überprüfung der korrekten Tarifanwendung durch den einzelnen Beschäftigten - mit den hinlänglich bekannten rechtlichen und tatsächlichen Schwierigkeiten.

\section{Tarifvertragsinhalte}

Die bisher abgeschlossenen Tarifverträge über Branchenzuschläge folgen grundsätzlich derselben Systematik: Gestaffelt nach Dauer des Einsatzes im Kundenbetrieb wird ein $\mathrm{Zu}$ schlag gezahlt, der sich aus dem Stundenentgelt der BAP/ BZA- bzw. iGZ-Entgelttarifverträge errechnet. Die Tarifverträge der Branchen unterscheiden sich dabei in den jeweiligen Prozentwerten der einzelnen Zuschlagsstufen. Darin kommt allerdings keine unterschiedliche Wertigkeit zum Ausdruck, sondern es werden hierdurch die unterschiedlichen Tarifentgelte der Branchen berücksichtigt: Zusammen mit der Grundvergütung der Entgelttarifverträge Leiharbeit soll der Zuschlag die Bezahlung der Beschäftigten an das jeweilige Vergütungsniveau heranführen. Die Höhe der Branchenzuschläge wurde deshalb gesondert für jede Branche anhand der durchschnittlichen vorhandenen Tarifentgelte (Grundentgelte und gegebenenfalls vorhandene Leistungsbestandteile) festgelegt.

6 Vgl. BAG vom 14.12.2010-1 ABR 19/10 und vom 13.03.2013-5 AZR 954/11 u.a. sowie die Pressemeldung des BAP vom 05.02.2013.

7 Vgl. Bundesagentur für Arbeit (2013): Der Arbeitsmarkt in Deutschland, Zeitarbeit in Deutschland - Aktuelle Entwicklungen, Nürnberg, S. 6.

8 Vgl. z. B. LAG Niedersachsen vom 19.09.2012-17 TaBV 124/11; LAG Berlin-Brandenburg vom 20.12.2012-4TaBV 1163/12 und 09.01.2013-15 Sa 1635/12 zur Mitbestimmung bei Dauereinsätzen.

9 Vgl. BAG vom 25.01.2005-1 ABR 61/03, wonach das Mitbestimmungsrecht bei Einstellungen kein Instrument zur Vertragsinhaltskontrolle darstellt.

10 Vgl. Krause, D. (2012): Neue tarifvertragliche Regeln für die Leiharbeit in der Metallindustrie, in: Neue Zeitschrift für Arbeitsrecht (NZA) 29 (15), S. 835-836; ArbG Braunschweig vom 22.02.2013-4 BV 14/12 (nicht rechtskräftig). 
Der Tarifvertrag findet in allen Betrieben der jeweiligen Branche Anwendung, auf die Tarifgebundenheit des Entleihbetriebes kommt es damit nicht an. Die Definition der einzelnen Branchen lehnt sich teilweise an die Satzungsbestimmungen der jeweiligen Gewerkschaften an. Dies bringt natürlich einige Überschneidungen und Unschärfen mit sich, die im Einzelfall aufgelöst werden müssen. Im Zweifel richtet sich die Einordnung nach dem im Entleihbetrieb angewandten Tarifvertrag.

Der Branchenzuschlag wird für die Dauer des Einsatzes im Kundenbetrieb gezahlt und richtet sich nach der jeweiligen ununterbrochenen Einsatzdauer. Unterbrechungszeiten von weniger als drei Monaten bleiben unberücksichtigt. Die höchste Zuschlagsstufe wird in allen Tarifverträgen nach einem Einsatz von neun Monaten im gleichen Einsatzbetrieb erreicht. Die Festlegung der zeitlichen Stufen hat für Kritik gesorgt, insbesondere mit Hinweis auf die durchschnittliche Beschäftigungsdauer in der Leiharbeit. Nach den Zahlen der Bundesagentur liegt diese für knapp die Hälfte der Arbeitsverhältnisse unter drei Monaten. Allerdings erfasst diese nur die Beschäftigungsdauer beendeter Arbeitsverhältnisse. ${ }^{11}$ Nach Einschätzung der IG Metall ist eine solche Stufenregelung vertretbar, da in der Metall- und Elektroindustrie das Gros der Leiharbeitsbeschäftigten tatsächlich längerfristig eingesetzt wird. ${ }^{12}$

Bestehen in Einsatzbetrieben bereits Regelungen, die eine Verpflichtung des Entleihers zu einer besseren Bezahlung von Leiharbeitsbeschäftigten vorsehen, so werden diese durch die Tarifverträge über Branchenzuschläge nicht tangiert. Genauso wenig entfällt hierdurch der tarifliche Anspruch auf Branchenzuschläge. Der Beschäftigte hat Anspruch auf die jeweils günstigere Regelung.

\section{Versuche der Umgehung}

Das Einkommensplus aufseiten der Beschäftigten bedeutet Kostensteigerungen für die Arbeitgeber. Schon im Vorfeld des Inkrafttretens der ersten Tarifverträge am 1. November 2012 hat es Hinweise gegeben, dass es zu Versuchen kommen könnte, die Zahlung der Branchenzuschläge zu umgehen. Das tatsächliche Ausmaß ist auch zum jetzigen Zeitpunkt sehr schwierig zu ermitteln und zu verifizieren. Viele betroffene Leiharbeitsbeschäftigte schildern der IG Metall zwar ihre negativen Erfahrungen, möchten häufig aber wegen zu befürchtender Sanktionen weder ihre Firma noch den Einsatzbetrieb nennen.

Eine Umfrage der IG Metall unter Leiharbeitsbeschäftigten Anfang 2013 hat ergeben, dass an $42 \%$ der Befragten bereits Branchenzuschläge ausbezahlt wurden. ${ }^{13} 23 \%$ sind sich nicht sicher, ob sie die Zuschläge erhalten - ein deutliches Spiegelbild der unübersichtlichen Abrechnungsmodalitäten in der Branche. Von den $35 \%$ der Befragten, die keine Zuschläge erhalten haben, gab knapp die Hälfte (45 \%) an, dass diese mit übertariflichen Zulagen oder zusätzlichen Leistungen aus betrieblichen Regelungen in den Einsatzbetrieben verrechnet wurden. Letzteres ist auch Ausdruck dafür, dass in der Metall- und Elektroindustrie in vielen Betrieben bereits bessere Vergütungsregelungen durchgesetzt werden konnten.

Die Ergebnisse der Umfrage, aber auch die Anfragen und Beratungen in den IG Metall-Verwaltungsstellen vor Ort ergeben insgesamt eine Reihe typischer Umgehungstatbestände, die an einzelnen Regelungen des Tarifvertrages festgemacht werden können.

Die Tarifverträge über Branchenzuschläge enthalten eine Besitzstandsregelung, ermöglichen jedoch eine Verrechnung des gezahlten Branchenzuschlags mit übertariflichen Leistungen. An sich eine unproblematische Regelung, deren Umsetzung jedoch ein sehr anschauliches Bild bestimmter Praktiken in der Branche bietet. Bereits vor Inkrafttreten des Tarifvertrages wurden den Beschäftigten nämlich in großem Maßstab Änderungsverträge zur Unterschrift vorgelegt. Diese enthalten standardmäßig Klauseln, die nicht nur die Möglichkeit der Anrechnung von übertariflichen Zulagen vorsehen, sondern ganz pauschal auch sämtliche außertariflichen Leistungen, z. B. auch Erschwernis- und Schmutzzulagen und sogar Aufwandsersatzleistungen und Fahrgelder zur Anrechnung bringen sollen. Begründet wird dies von Arbeitgebern mit dem abwegigen Argument, diese Leistungen seien in Wirklichkeit Entgeltleistungen mit lediglich „anderer Bezeichnung “. Hier zeigt sich eine Praxis, dass steuer- und sozialversicherungsprivilegierte Aufwandsersatzleistungen und Fahrgelder als „Kompensation“ anstelle einer korrekten Eingruppierung gezahlt werden. Teilweise wurden die Beschäftigten auch einfach um ein oder zwei Entgeltgruppen abgruppiert, um, bei gleichbleibender Gesamtvergütung, den übertariflichen Anteil zu erhöhen. Der Arbeitgeber erhält so zusätzliche Spielräume, um Branchenzuschläge zu verrechnen. An dieser Stelle wird auch die Schwäche eines eigenen Eingruppierungssystems für eine weitgehend betriebsratslose Branche deutlich.

Eine weitere Umgehungsmöglichkeit ergibt sich aus dem Branchenbezug der Zuschlagsregelungen. Zum einen wird mitunter einfach die Zugehörigkeit zu einer zuschlagspflichtigen Branche infrage gestellt bzw. eine Branche mit einem "günstigeren“ Zuschlag reklamiert. Konflikte hat es in der Metall- und Elektroindustrie insbesondere bei Automobilzulieferern gegeben. Die Regelung, wonach im Zweifelsfall der angewandte Tarifvertrag für die zugehörige Branche maßgeblich ist, dürfte diese Praxis aber auf Betriebe begrenzen, die nie einen Tarifvertrag angewandt haben und in denen eine Gewerkschaft nicht vertreten ist.

11 Vgl. Bundesagentur für Arbeit (Fn. 7), Seite 17.

12 Eine Befragung von Leiharbeitsbeschäftigten durch die IG Metall Ende 2011 ergab u.a., dass die Einsatzdauer bei $22 \%$ der Beschäftigten zwischen 6 und 12 Monaten, bei $55 \%$ der Beschäftigten sogar über 12 Monate beträgt.

13 IG Metall, Report Branchenzuschläge (erscheint im Juni 2013). 
Die zeitliche Staffelung des Branchenzuschlags ist einer möglichen Umgehungsstrategie durch gezieltes Abziehen der Beschäftigten vor Erreichen der nächsthöheren Stufe ausgesetzt. Nur wenn Beschäftigte vor Ablauf von drei Monaten in den gleichen Betrieb zurückkehren, behalten sie ihren bisher erworbenen Anspruch. Die Ankündigung der Branche, die tariflichen Regelungen würden zu einer „höheren Dynamik“ im Einsatz von Leiharbeitskräften führen sowie einzelne Mitteilungen von Leiharbeitsbeschäftigten, sie seien „passgenau“ vor Erreichen der nächsten Stufe abgezogen worden, lassen befürchten, dass ein systematischer Austausch im Rahmen „rollierender Einsätze“ Anwendung finden könnte. Konkrete Anhaltspunkte gibt es hierfür allerdings derzeit nicht. Die Mitbestimmung der Betriebsräte im Einsatzbetrieb kann aber einen solchen missbräuchlichen Austausch einschränken.

Die weitaus größte Gefahr für die neuen tariflichen Regelungen besteht allerdings darin, dass diese im Rahmen von Werkvertragsverhältnissen umgangen werden. Zwei Varianten sind bislang aufgetreten. Zum einen setzen viele Personaldienstleister ihre Beschäftigten nicht nur im Rahmen der Arbeitnehmerüberlassung ein, sondern auch als Werkvertragsnehmer. In den Arbeitsverträgen dieser Beschäftigten finden sich Klauseln, nach denen sie sich auch dazu bereit erklären, im Rahmen eines Werkvertrages eingesetzt zu werden. Die Tarifverträge über Branchenzuschläge gelten allerdings nur bei Arbeitnehmerüberlassung. ${ }^{14}$ Häufig ist nicht einmal für die Beschäftigten erkennbar, auf welcher vertraglichen Grundlage der Einsatz erfolgt. Vergleichbar sind Konstruktionen, in denen Beschäftigte zu eigenständigen Tochterunternehmen verschoben werden. Erfolgt ein solcher Wechsel der Vertragsgrundlage im gleichen Einsatzbetrieb und ohne Veränderung der konkreten Rahmenbedingungen, bestehen starke Anhaltspunkte für einen Scheinwerkvertrag.

Zum anderen ist folgende Konstellation zu beobachten: Der Beschäftigte wird an einen Betrieb verliehen, der nicht dem Tarifvertrag unterliegt (z. B. ein Handwerksbetrieb). Er wird von diesem Betrieb dann im Rahmen eines Werkvertragsverhältnisses in einem an sich branchenzuschlagspflich-

14 Es läge nahe, eine solche Konstruktion auf ihre AGB-Konformität zu prüfen: Von den Tarifverträgen über Branchenzuschläge wird der Beschäftigte bei solchen Tätigkeiten nicht erfasst, der Arbeitgeber kann somit einseitig bestimmen, welche Bedingungen im Arbeitsverhältnis Anwendung finden.

15 „Freie Industriedienstleistung als Alternative zur regulierten Zeitarbeit“ lautete das Thema derTagung des "Zentrums für Arbeitsbeziehungen und Arbeitsrecht" vom 09.09.2011.

16 Vgl. u. a. Zeit online vom 08.12.2011, Spiegel online vom 26.02.2013, die öffentliche Anhörung im BT-Ausschuss für Arbeit und Soziales vom 23.04.2012 und das Symposium Werkverträge des BMAS vom 11.03.2013 sowie die Gesetzentwürfe von Linken und SPD (BT-Drucksachen 17/12373 u. 17/12378).

17 Zur Initiative der IG Metall siehe: http://www.fokus-werkvertraege.de/ tigen Industriebetrieb eingesetzt. Der Beschäftigte hat hier keinen Anspruch auf einen Branchenzuschlag. Selbst wenn solche Fälle nicht gegen den Wortlaut des Tarifvertrages verstoßen: Es spricht vieles für ein bewusstes Umgehen der Branchenzuschläge, wenn es sich bei dem Entleiher um einen "Strohmann" handelt, der ohne eigenständigen Betriebszweck ausschließlich auf Basis dieser Vertragskonstruktion agiert. Solche Werkvertragsverhältnisse stellen für die Tarifverträge über Branchenzuschläge die größte offene Flanke dar. Nicht nur haben zahlreiche Verleihfirmen Tochterunternehmen gegründet, die „Werkvertragslösungen“ anbieten. Werkverträge werden sogar offen als „Alternative zur regulierten Zeitarbeit“15 propagiert. Da nicht nur die Branchenzuschläge, sondern in der Praxis sogar die Lohnuntergrenzen in der Leiharbeit (derzeit 8,19 €/West; 7,50 €/Ost) unterlaufen werden, ist das Thema "Scheinwerkverträge“ inzwischen längst in der Öffentlichkeit angekommen. ${ }^{16}$

\section{Ein vorläufiges Fazit}

Sechs Monate nach Inkrafttreten der ersten Tarifverträge über Branchenzuschläge ist es sicherlich noch zu früh, eine Bilanz zu ziehen. Umgehungsversuche sind in nicht unerheblicher Zahl festzustellen, dennoch kann man aus diesen zum jetzigen Zeitpunkt nicht auf eine großflächige und einheitliche Strategie des Unterlaufens der Tarifverträge schließen. Angesichts der schwierigen Gegebenheiten in der Leiharbeit mit der Vielzahl von nichtverbandsgebundenen Tarifanwendern und unzureichenden gewerkschaftlichen Strukturen in den Verleihbetrieben ist es schwierig, sich ein klares Bild zu machen. Umso wichtiger ist es, dass die Gewerkschaften die Leiharbeit als betriebs- und tarifpolitisches Handlungsfeld weiter im Fokus behalten.

Von der bewussten oder unbewussten fehlerhaften Anwendung der Tarifverträge scharf zu unterscheiden ist dagegen die Ausweichstrategie zu Werkvertragsverhältnissen. Hier ergibt sich die Notwendigkeit, auch neuen Formen des Lohndumpings durch entschiedenes gewerkschaftliches Handeln auf allen Ebenen entgegenzutreten. ${ }^{17}$

\section{AUTOREN}

HELGA SCHWITZER, geschäftsführendes Vorstandsmitglied der IG Metall, zuständig für den Bereich Tarifpolitik.

helga.schwitzer@igmetall.de

DIRK SCHUMANN, Sekretär beim IG-Metall-Vorstand, Funktionsbereich Tarifpolitik.

dirk.schumann@igmetall.de 